Pacific Journal of Mathematics

MONOTONE DECOMPOSITIONS OF IRREDUCIBLE 


\title{
MONOTONE DECOMPOSITIONS OF IRREDUCIBLE HAUSDORFF CONTINUA
}

\author{
G. R. GORDH, JR.
}

It is shown that a number of important results concerning irreducible metric continua can be generalized to (nonmetric) irreducible continua. For example, if $M$ is a (nonmetric) continuum which is irreducible between a pair of points and which contains no indecomposable subcontinuum with interior, then there exists a monotone continuous map of $M$ onto a generalized arc, such that each point inverse has void interior. This result is applied to a study of hereditarily unicoherent, hereditarily decomposable continua. Certain properties of trees follow as corollaries. Also, trees are characterized as inverse limits of monotone inverse systems of dendrites.

In recent years there has been a growing interest in the study of (nonmetric) continua. It is well known (e.g., [6]) that some of the most useful and important properties of metric continua do not hold for (nonmetric) continua. It is the purpose of this paper to indicate that a substantial number of theorems concerning irreducible metric continua can be generalized to irreducible continua. These results are then applied to a study of certain hereditarily unicoherent continua.

In particular, $\S 2$ contains generalizations of many of the results about irreducible metric continua appearing in Chapter 1 of [11]. These results are applied in $\S 3$ to obtain generalizations of a number of theorems due to Miller [8] concerning hereditarily unicoherent continua. Section 4 contains several results about trees which follow as corollaries of theorems in $\S 3$. Also, it is proved that every tree can be written as a monotone inverse limit of dendrites. In Chapter 2 of [11], Thomas discusses metric continua which are hereditarily of type $A^{\prime}$. His definition is extended, in $\S 5$, to (nonmetric) continua and several characterizations of such continua are obtained.

The reader is referred to [3], [5], and [14] for general results concerning continua (i.e., compact, connected Hausdorff spaces). It will be necessary to refer to results which are stated in the literature for metric continua; however, this will be done only when the proof for continua is essentially the same as that for metric continua.

The author is indebted to Professor F. Burton Jones for his advice and encouragement in the preparation of this paper. 
2. Continua of type A. We observe that Theorem 1 and Theorem 7 of [11, Chapter 1] are true, as stated, for (non-metric) continua. To prove Theorem 1, apply [9, Theorem 47, page 16] to the proof as given in [11].

Let $M$ be a continuum which is irreducible between a pair of points $x$ and $y$. A decomposition $\mathscr{D}$ of $M$ is said to be admissible in case each element of $\mathscr{D}$ is a nonvoid proper subcontinuum of $M$, and each element of $\mathscr{D}$ which does not contain $x$ or $y$ separates $M$. Notice that an admissible decomposition is not required by definition to be upper semi-continuous. However, we will show that an admissible decomposition must, in fact, be upper semi-continuous. Thus, for metric continua, our definition is equivalent to the definition in [11].

A generalized arc is a continuum $A$ with precisely two nonseparating points. It is well known that $A$ can be totally ordered in such a way that the order topology and the original topology coincide. We will frequently denote $A$ by $[a, b]$ where $a$ and $b$ are the nonseparating points of $A$.

TheOREM 2.1. Let $M$ denote a continuum. Let $\mathscr{D}=\{D(x)\}$ be a decomposition of $M$ such that (1) for each $x \in M, D(x)$ is a proper subcontinuum of $M$, and (2) there exist elements $D(a)$ and $D(b)$ of $\mathscr{D}$ such that every element $D(x)$ of $\mathscr{D}$ distinct from $D(a)$ and $D(b)$ separates $D(a)$ from $D(b)$. Then $\mathscr{D}$ is an upper semi-continuous decomposition, and $M / \mathscr{D}$ is a generalized arc.

Proof. For each $x$ in $M-[D(a)+D(b)], M-D(x)=A_{x}+B_{x}$ where $a \in A_{x}, b \in B_{x}$, and $A_{x}$ and $B_{x}$ are connected. If $x$ and $y$ are in $M-[D(a)+D(b)]$ and $D(x) \neq D(y)$, then $D(y) \subset A_{x}$ if and only if $A_{y} \subset A_{x}$; also $D(y) \subset B_{x}$ if and only if $B_{y} \subset B_{x}$. Define $D(x)<(D(y)$ whenever $A_{x} \subset A_{y}$, and let $D(a)<D(z)<D(b)$ for all $z$ in

$$
M-[D(a)+D(b)] \text {. }
$$

Then $<$ is a total order on $\mathscr{D}$. If $f: M \rightarrow \mathscr{D}$ denotes the natural map, then it is readily seen that $f$ is continuous with respect to the order topology on $\mathscr{D}$. The conclusion of the theorem now follows.

COROLlary 2.1. Let $M$ be a continuum which is irreducible from $x$ to $y$. If $\mathscr{D}$ is an admissible decomposition for $M$, then $\mathscr{D}$ is upper semi-continuous and $M / \mathscr{D}$ is a generalized arc.

A continuum $M$ is of type $A$ provided that it is irreducible between a pair of points and has an admissible decomposition; $M$ is of type $A^{\prime}$ if it is of type $A$ and has an admissible decomposition each of whose elements has void interior. 
THEOREM 2.2. Let $M$ be a continuum irreducible from $x$ to $y$. If $M$ has an admissible decomposition, then it has one which is minimal (with respect to partial order by refinement).

Proof. See the proof of [11, Theorem 3, page 8]. Notice that we are not required to prove the upper semi-continuity of the decomposition.

Suppose that $M$ is a continuum irreducible between two points. If $M$ is of type $A$, let $\Delta$ denote the collection of all admissible decompositions of $M$. For each $\mathscr{D} \in \Delta$, let $f: M \rightarrow M / \mathscr{D}$ denote the natural map. Thus $f$ is a continuous monotone function from $M$ onto a generalized arc. Observe that every monotone map from $M$ onto a generalized arc is obtained in this manner.

THEOREM 2.3. Let $M$ be a continuum of type $A, \mathscr{D} \in \Delta$, and $f: M \rightarrow M / \mathscr{D}$. Suppose that $K$ is a subcontinuum of $M$ such that $f(K)=[r, s]$ where $[r, s]$ is a nondegenerate subinterval of $M / \mathscr{D}$. Then $f^{-1}(r) \cap K$ and $f^{-1}(s) \cap K$ are continua, and for $r<t<s, f^{-1}(t)$ is contained in and separates $K$. In particular $\left.f\right|_{K}$ is a monotone map of $K$ onto $[r, s]$; thus, if $K$ is irreducible, $K$ is of type $A$.

Proof. Suppose that $M$ is irreducible from $x$ to $y$ and $M / \mathscr{D}=$ $[a, b]$. If $r<t<s$, then $f^{-1}(t) \subset K$; for if $p$ is in $f^{-1}(t)-K$ then $f^{-1}([a, r])+K+f^{-1}([s, b])$ is a proper subcontinuum containing $x$ and $y$. Clearly $f^{-1}(t)$ separates $K$, since it separates $M$. To see that $f^{-1}(r) \cap K$ is connected, let $K^{\prime}=\cap\left\{c l\left[f^{-1}((r, u))\right] ; u \in(r, s)\right\}$. Then $K^{\prime}$ is a subcontinuum of $f^{-1}(r) \cap K$ which is easily seen to intersect each component of $f^{-1}(r) \cap K$. Thus $f^{-1}(r) \cap K$, as well as $f^{-1}(s) \cap K$, is connected.

THEOREM 2.4. Let $M$ be a continuum of type $A$; then $\triangle$ contains a unique minimal element.

Proof. The proof of [11, Theorem 6, page 10] is valid, since we are not concerned with proving the upper semi-continuity of the decomposition.

Corollary 2.2. Let $M$ be a continuum of type $A^{\prime}$. If $\mathscr{D} \in \Delta$ is such that each element of $\mathscr{D}$ has void interior, then $\mathscr{D}$ is the minimal element of $\Delta$.

Proof. Suppose that $\mathscr{D}^{\prime} \in \Delta$ such that $\mathscr{D}^{\prime} \leqq \mathscr{D}$. Let $D(\alpha)$ and $D(b)$ denote the nonseparating elements of $\mathscr{D}$. Then $M-D(a)$ is connected, and since $D(a)^{0}=\varnothing,[M-D(a)]+D^{\prime}(\alpha)$ is connected. Thus 
$D(a)=D^{\prime}(a)$ and $D(b)=D^{\prime}(b)$. Given $x$ in $M-[D(a)+D(b)]$, write $M-D(x)=A_{x}+B_{x}$ uniquely. Then $M=\bar{A}_{x}+\bar{B}_{x}$ and

$$
\varnothing \neq \bar{A}_{x} \cap \bar{B}_{x} \subset D(x) \text {. }
$$

Given $z$ in $D(x), D^{\prime}(z)$ must separate $D(a)$ from $D(b)$; thus $\bar{A}_{x} \cap \bar{B}_{x} \subset$ $D^{\prime}(z)$. Consequently, $D^{\prime}(x)=D(x)$ and $\mathscr{D}^{\prime}=\mathscr{D}$.

The following useful result is a generalization of [11, Theorem 8, page 14].

THEOREM 2.5. Let $M$ be a continuum of type $A, \mathscr{D} \in \Delta$, and $f: M \rightarrow M / \mathscr{D}=[a, b]$. Then for $a \leqq r<s \leqq b, \quad c l\left[f^{-1}((r, s))\right]=K$ is a subcontinuum of $M$ which is irreducible from every point of

$$
K \cap f^{-1}(r)=K_{r}
$$

to every point of $K \cap f^{-1}(s)=K_{s}$. Also $K_{r}$ and $K_{s}$ are subcontinua of $K$ with void interior relative to $K$.

Proof. Since $K_{r} \subset K-f^{-1}((r, s)), K_{r}^{0}=\varnothing$. By Theorem 2.3, $K_{r}$ and $K_{s}$ are subcontinua of $K$. That $K$ is irreducible from $K_{r}$ to $K_{s}$ follows from the proof of [11, Theorem 8, page 14].

THEOREM 2.6. Let $M$ denote a continuum which is irreducible between two closed subsets $H$ and $K$ such that every subcontinuum of $M$ with nonvoid interior is decomposable. Then the following hold. (a) There is a decomposition of $M, M=M_{H}+M_{K}$, where $H \subset M_{H}$, $K \subset M_{K}$ and $\operatorname{cl}\left[M_{H}-M_{K}\right] \cap M_{K}$ is connected. (b) If $U$ and $V$ are open subsets of $M$ such that $H \subset U \subset \bar{U} \subset V \subset M-K$ and both $\partial U$ and $\partial V$ are connected, then there is an open set $W$ of $M$ such that $\bar{U} \subset W \subset$ $\bar{W} \subset V$ and $\partial W$ is connected.

Proof. The proof in [11, Theorem 9, page 14] is valid. Note that we have added the hypothesis that $\partial U$ is connected in part (b).

THEJFEM 2.7. Let $M$ be a continuum irreducible between a pair of points $x$ and $y$. A necessary and sufficient condition that $M$ be of type $A^{\prime}$ is that every subcontinuum of $M$ with nonvoid interior be decomposable.

Proof of sufficiency. Using the construction in [11, Theorem 10, page 15] we define a monotone function $f: M \rightarrow[0,1]$. According to [4, Lemma 3, page 114] $f$ is continuous. Thus $\left\{f^{-1}(t) ; t \in[0,1]\right\}$ is an admissible decomposition for $M$. According to Theorem 2.2 there exists a minimal admissible decomposition for $M$, say $\mathscr{D}$. If some ele- 
ment $D \in \mathscr{D}$ has nonvoid interior then $\operatorname{cl}\left(D^{0}\right)$ is of type $A$. Let $\mathscr{D}^{\prime}$ denote an admissible decomposition for $\operatorname{cl}\left(D^{\circ}\right)$. By combining $\mathscr{D}$ and $\mathscr{D}^{\prime}$ in the natural way, one obtains an admissible decomposition which refines $\mathscr{D}$ properly. Thus no element of $\mathscr{D}$ has nonvoid interior, and $M$ is of type $A^{\prime}$.

Proof of necessity. See [11, Theorem 10, page 16].

By making the obvious necessary modifications, one can also generalize Theorems 17 through 22 of Chapter 1 of [11]. As in [11] we define $K(z)=\{y \in M ; M$ is nonaposyndetic at $z$ with respect to $y\}$ and $L(z)=\{y \in M ; M$ is nonaposyndetic at $y$ with respect to $z\}$. Observe that $L(z)=T(z)$ where $T$ denotes the set function in [2]. The statements and proofs of Theorems 18 and 19 can be shortened by observing that $K(z)=L(z)$ for any point $z$ of an irreducible continuum [2, Theorem 2, page 116]. Since Theorem 19 provides a concise topological characterization for continua of type $A^{\prime}$, we include its statement as Theorem 2.8 .

THEOREM 2.8. Let $M$ denote a continuum irreducible from $x$ to $y$. Then $M$ is of type $A^{\prime}$ if and only if $K(z)^{0}=\varnothing$ for each $z$ in $M$.

3. Hereditarily unicoherent, hereditarily decomposable continua. In [8] Miller proves that every irreducible, hereditarily decomposable metric continuum is of type $A$ (this is a corollary of our Theorem 2.7). By applying this result she obtains a number of conditions which imply that a hereditarily decomposable metric continuum is hereditarily unicoherent, and she also shows that hereditarily unicoherent, hereditarily decomposable metric continua have certain properties analogous to properties of acyclic continuous curves (i.e., dendrites). In this section we will apply Theorem 2.7 to show that most (but not all) of Miller's results can be generalized to (nonmetric) continua.

It is easy to see that a continuum $M$ is hereditarily unicoherent if and only if for each pair of distinct points $x$ and $y$ of $M$ there exists exactly one subcontinuum of $M$ which is irreducible from $x$ to $y$.

By a generalized simple closed curve we mean a continuum which is separated by the omission of any two of its points. A point $p$ is said to cut the continuum $M$ in case there exist points $x$ and $y$ in $M$ such that each subcontinuum of $M$ containing $x$ and $y$ also contains $p$. Such a point, $p$, is said to cut $x$ from $y$ in $M$, or to cut between $x$ and $y$ in $M$.

The theorems that follow extend and generalize (to nonmetric continua) Theorems 2.4 through 2.9 of [8].

THEOREM 3.1. Let $M$ be a continuum of type $A$, and $\mathscr{D} \in \Delta$. If 
each element of $\mathscr{D}$ is unicoherent then $M$ is unicoherent.

Proof. Let $f: M \rightarrow M / \mathscr{D}=[a, b]$ denote the natural map. Suppose that $H$ and $K$ are proper subcontinua of $M$ such that $M=H+$ $K$. If $f(H)=[a, c]$ and $f(K)=[c, b]$ then $H \cap K \subset f^{-1}(c)$. Now

$$
\left[H \cap f^{-1}(c)\right]+\left[K \cap f^{-1}(c)\right]=f^{-1}(c) .
$$

Since $H \cap f^{-1}(c)$ and $K \cap f^{-1}(c)$ are continua (Theorem 2.3), and $f^{-1}(c)$ is unicoherent, $H \cap K=\left[H \cap f^{-1}(c)\right] \cap\left[K \cap f^{-1}(c)\right]$ is connected. The other cases are handled in a similar manner, although they do not depend on the unicoherence of the elements of $\mathscr{D}$.

THEOREM 3.2. Let $M$ be a continuum of type $A$, and $\mathscr{D} \in \Delta$. If $f: M \rightarrow M / \mathscr{D}=[a, b]$ is an open map, then $M$ is unicoherent.

Proof. Let $H$ and $K$ be proper subcontinua of $M$ such that $M=$ $H+K$. If $f(H)=[a, c]$ and $f(K)=[c, b]$ then

$$
H \cap f^{-1}(c)=f^{-1}(c)=K \cap f^{-1}(c)
$$

since $f$ is open. Thus $H \cap K=f^{-1}(c)$ which is connected. The other cases are handled as in Theorem 3.1.

THEOREM 3.3. If $M$ is a hereditarily decomposable continuum which is not unicoherent, then $M$ contains a continuum $N$ which is a generalized simple closed curve with respect to the elements of a monotone upper semi-continuous decomposition $\mathscr{D}$. Furthermore, if $D_{1}$ and $D_{2}$ are in $\mathscr{D}$ then $N-\left(D_{1}+D_{2}\right)=U+V$ where $U$ and $V$ are disjoint connected open sets such that (1) $N=\bar{U}+\bar{V}$, (2) $\bar{U}$ and $\bar{V}$ are irreducible from $D_{1}$ to $D_{2}$, and (3) any subcontinuum of $D_{1}+D_{2}+$ $U$ which intersects $D_{1}$ and $D_{2}$ contains $\bar{U}$.

Proof. Apply Theorem 2.7 to the proof of [8, Theorem 2.6, page 187].

THeorem 3.4. Let $M$ be a hereditarily decomposable continuum. $M$ is hereditarily unicoherent if and only if $M$ contains no subcontinuum $N$ which is a generalized simple closed curve with respect to the elements of a monotone upper semi-continuous decomposition.

Proof. If $M$ is not hereditarily unicoherent, apply Theorem 3.3. Conversely, suppose that $f: N \rightarrow C$, where $N$ is a subcontinuum of $M$, $f$ is monotone and onto, and $C$ is a generalized simple closed curve. Write $C=A+B$ where $A$ and $B$ are generalized arcs. Then $f^{-1}(A) \cap f^{-1}(B)$ is not connected. 
THEOREM 3.5. Let $M$ be a hereditarily decomposable continuum. Suppose that there exists a cardinal number $k \leqq c$ such that given $k$ points of $M$ there exists one of them which cuts between some pair of them. Then $M$ is hereditarily unicoherent.

Proof. Suppose $M$ is not hereditarily unicoherent. According to Theorem 3.4 there exists a subcontinuum $N$ of $M$, a generalized simple closed curve $C$, and a monotone map $f$ from $N$ onto $C$. Choosing $k$ distinct points of $C$ it is clear that no one cuts between any pair of them. The theorem follows.

THEorem 3.6. If $M$ is a hereditarily decomposable continuum every subcontinuum of which is irredusible about a closed proper subset having only countably many components, then $M$ is hereditarily unicoherent.

Proof. Apply [5, Theorem 6, page 173] to the proof of [8, Theorem 2.9].

Theorem 3.6 does not remain true if "countably many components" is replaced by "c components". A simple modification of Example 2 [11, page 12] produces a metric continuum which is irreducible about a closed set with uncountably many components and is not unicoherent.

In order to obtain generalizations of theorems in [8, Section 3, page 190] we prove a generalization of a theorem due to R. L. Moore [10].

THEOREM 3.7. Let $M$ denote a hereditarily unicoherent continuum, and suppose that each indecomposable subcontinuum of $M$ is irreducible. If $H$ is an irreducible subcontinuum of $M$ then $H$ is contained in a maximal irreducible subcontinuum.

Proof. Throughout this proof $\langle x, y\rangle$ denotes the unique irreducible continuum from $x$ to $y$.

Suppose that $H$ is irreducible from $a$ to $b$. Let $\left\{H_{\alpha}\right\}$ be a maximal monotonic collection of continua such that $H \subset H_{\alpha}$ for each $\alpha$, and $H_{\alpha}=\left\langle\alpha, h_{\alpha}\right\rangle$ for some $h_{\alpha}$ in $M$. Let $K=\operatorname{cl}\left(\mathbf{U}_{\alpha} H_{\alpha}\right)$. We will prove that the continuum $K$ is irreducible from $a$ to some point $k$. Assume not. Observe that if $A$ is a proper subcontinuum of $K$ which contains $a$, then $K-A$ is connected. There are two cases to consider.

Case 1. Suppose that $\operatorname{cl}(K-A)$ is indecomposable for some subcontinuum $A$ of $K$ which contains $a$. Let $T=\operatorname{cl}(K-A)$. Then $T \cap$ 
$A$ is a proper subcontinuum of $T$; hence $T \cap A$ is contained in a composant $C$ of $T$. Since $T$ is irreducible, it contains at least two composants. Choose $k \in T-C$. Then $\langle a, k\rangle=K$. To see this, suppose that $\langle a, k\rangle \neq K$. Then $\langle a, k\rangle \cap T$ is a continuum which intersects two composants of $T$; thus $T \subset\langle a, k\rangle$. Choose $h_{\alpha} \in A-\langle a, k\rangle, h_{\beta} \in K-A$. Then $H_{\alpha} \not \subset H_{\beta}$ and $H_{\beta} \not \subset H_{\alpha}$, which is a contradiction.

Case 2. $\operatorname{cl}(K-A)$ is decomposable for each subcontinuum $A$ of $K$ containing $a$. If

$$
\operatorname{cl}(K-A)=E+F
$$

is any decomposition of $\operatorname{cl}(K-A)$, then $A \cap F=\varnothing$ or $A \cap E=\varnothing$. Using this fact it is easy to verify that there exists an $H_{\beta}$ such that $A \subset H_{\beta}^{0}$. In particular, given an $H_{\alpha}$, there exists an $H_{\beta}$ such that $H_{\alpha} \subset H_{\beta}^{\nu}$. Choosing $k$ in $\bigcap_{\alpha} c l\left(K-H_{\alpha}\right)$ it follows that $\langle a, k\rangle=K$.

In either case, $K$ is "maximally irreducible" from $a$ to some point $k$. If $\langle x, y\rangle$ contains $K=\langle a, k\rangle$ properly, then $\langle x, y\rangle=\langle x, k\rangle$ or $\langle x, y\rangle=\langle y, k\rangle$. For suppose not and let $x \notin K$. Then $k \notin\langle a, x\rangle$; hence $y \notin\langle a, x\rangle$. Since $\langle x, k\rangle$ is properly contained in $\langle x, y\rangle, y \notin\langle x, k\rangle$. But $K \subset\langle a, x\rangle+\langle x, k\rangle$; thus $y \notin K$. Now $\langle x, y\rangle \subset\langle a, x\rangle+\langle a, y\rangle$ which misses $k$. This is a contradiction.

Let $L$ be a continuum containing $K$ which is "maximally irreducible" from $k$ to some point. Then $L$ is a maximal irreducible subcontinuum containing $H$. For if $L \subset\langle z, y\rangle$ then $K \subset\langle x, y\rangle$. According to the argument above we can assume that $\langle x, y\rangle=\langle x, k\rangle$. It follows immediately that $\langle x, y\rangle=L$.

COROLlary 3.1. Let $M$ denote a hereditarily unicoherent, hereditarily decomposable continuum. If $H$ is an irreducible subcontinuum of $M$, then $H$ is contained in a maximal irreducible subcontinuum.

CoRollary 3.2 (Moore). Let $M$ denote a hereditarily unicoherent metric continuum. If $H$ is an irreducible subcontinuum of $M$, then $H$ is contained in a maximal irreducible subcontinuum.

Proof. Every indecomposable metric continuum is irreducible.

As in [8], we define a point $p$ to be a terminal point of the continuum $M$ in case every irreducible subcontinuum of $M$ which contains $p$ is irreducible from $p$ to some point. By making use of Theorem 2.7 and Corollary 3.1 we obtain the following generalizations of theorems in [8, $\S 3$, page 190$]$. 
$M$ is either a terminal point or a cut point of $M$.

THEOREM 3.9. A continuum which is hereditarily unicoherent and hereditarily decomposable has at least two terminal points.

THEOREM 3.10. A continuum which is hereditarily unicoherent and hereditarily decomposable is irreducible about the set of all its terminal points.

THEOREM 3.11. If the continuum $M$ is hereditarily decomposable and $K$ is a subset of $M$ consisting of some of the terminal points of $M$, then $M-K$ is connected.

In $\S 4$ we will see that Theorem 3.7 of [8] does not generalize to nonmetric continua.

4. Some properties of trees. A continuum $M$ is said to be a tree [12] if and only if given two distinct points $p$ and $q$ of $M$, there exists a third point which separates $p$ from $q$. The point $p$ of a tree $M$ is said to be an end point of $M$ if and only if $p$ is a nonseparating point of every generalized arc containing $p$. It is known [12] that a continuum $M$ is a tree if and only if $M$ is locally connected and hereditarily unicoherent. If $M$ is a metric continuum then $M$ is a tree if and only if $M$ is a dendrite [13, (1.1), page 88]. In Theorem 4.1 we show that a number of familiar properties of dendrites are also shared by trees.

Theorem 4.1. Let $M$ denote a tree. Then (1) $M$ is connected by generalized arcs, (2) each point of $M$ is a separating point or an end point, (3) each generalized arc in $M$ is contained in a maximal generalized arc, (4) $M$ has at least two end points, (5) $M$ is irreducible about the set of all its end points, (6) if $K$ is a subset of the end points of $M$, then $M-K$ is connected.

Proof. Let $A$ be a subcontinuum of $M$ irreducible from $p$ to $q$. Since $M$ is hereditarily unicoherent, each point of $A-(p+q)$ cuts $p$ from $q$ in $M$; thus, since $M$ is locally connected, each point of $A$ $(p+q)$ actually separates $p$ from $q$ in $M$. Consequently, $A$ is a generalized arc. Since $M$ is hereditarily decomposable, properties (2) through (6) follow from Theorems 3.7 through 3.11.

For a metric continuum $M$ the following properties are equivalent [13, (1.1), page 88]: (a) $M$ is a tree, (b) $M$ is locally connected and contains no (generalized) simple closed curve, (c) every subcontinuum 
of $M$ contains uncountably many separating points of $M$.

For (nonmetric) continua we have seen that condition (a) implies conditions (b) and (c). However, neither of these implications can be reversed. Mardešic has shown [6] that there exists a locally connected continuum which contains no proper locally connected subcontinuum. This example clearly satisfies condition (b), but is not a tree. The following example satisfies condition (c) but not (a); and also shows that [8, Theorem 3.7, page 193] does not generalize to (nonmetric) continua.

EXAMPLE. Let $C$ denote a circle, and let $M=C \times[0,1]$. We define a basis $\mathscr{B}$ for the topology on $M$ as follows: $V$ is in $\mathscr{B}$ if and only if (1) $V=p \times(r, s),(2) V=p \times(r, 1]$, or (3)

$$
V=(U \times[0,1])-\bigcup_{i=1}^{n}\left\{p_{i} \times\left[q_{i}, 1\right]\right\},
$$

where $U$ is open in the usual topology for $C, p_{i}$ is in $U$, and $0<$ $q_{i}<1$. If $\mathscr{T}$ denotes the topology generated by $\mathscr{B}$ then $(M, \mathscr{T})$ is seen to be a (compact Hausdorff) continuum with the desired properties.

Finally, we give a characterization of trees in terms of inverse limits. For a discussion of inverse limits systems, see [1].

THEOREM 4.2. The continuum $M$ is a tree if and only if $M$ is homeomorphic to the inverse limit of a monotone inverse system $\left(D_{\alpha}\right.$, $\pi_{\alpha \beta}$, A) where each $D_{\alpha}$ is a (metric) dendrite.

Proof. According to [12] we must show that $M$ is locally connected and hereditarily unicoherent. $M$ is locally connected by [1, Theorem 4.3, page 241]. A simple application of [1, page 235, 2.9] shows that $M$ is hereditarily unicoherent. On the other hand, since $M$ is locally connected, $M$ can be written as the inverse limit of a monotone inverse system $\left(D_{\alpha}, \pi_{\alpha \beta}, \Lambda\right)$ where each $D_{\alpha}$ is a locally connected metric continuum [7]. According to [1], $\pi_{\alpha}: M \rightarrow D_{\alpha}$ is monotone. It follows easily that $D_{\alpha}$ is a tree, hence a dendrite.

5. Continua hereditarily of type $A^{\prime}$. As in Chapter 2 of [11], we define a continuum $M$ to be hereditarily of type $A^{\prime}$ if and only if every nondegenerate subcontinuum of $M$ is of type $A^{\prime}$. If $M$ is a hereditarily decomposable metric continuum then $M$ is hereditarily of type $A^{\prime}$ if and only if $M$ is snake-like [11, Theorem 13, page 50]. In this section we obtain several topological characterizations of (nonmetric) continua which are hereditarily of type $A^{\prime}$. 
THEOREM 5.1. If the continuum $M$ is hereditarily of type $A^{\prime}$, then $M$ is hereditarily unicoherent and atriodic.

Proof. The proof of [11, Theorem 6, page 41] is valid for (nonmetric) continua.

LEMma 5.1. If the continuum $M$ is hereditarily unicoherent and atriodic, then given three points of $M$, one cuts between the other two.

THEOREM 5.2. The continuum $M$ is hereditarily of type $A^{\prime}$ if and only if $M$ is hereditarily unicoherent, hereditarily decomposable, and atriodic.

Proof. Suppose that $M$ is hereditarily unicoherent, hereditarily decomposable, and atriodic. According to Theorem 2.7 it suffices to show that every subcontinuum $N$ of $M$ is irreducible. Let $A$ be a maximal irreducible subcontinuum of $N$ (Theorem 3.7) which is irreducible from $p$ to $q$. If there exists a point $r$ in $N-A$ then, since $A$ is maximal irreducible, it follows that none of $p, q$, and $r$ cuts between the other two. This contradicts Lemma 5.1; hence $N=A$. The converse follows from Theorem 5.1.

THEOREM 5.3. Let $M$ denote a hereditarily decomposable continuum. Then $M$ is hereditarily of type $A^{\prime}$ if and only if given any three points of $M$ one cuts between the other two.

Proof. If $M$ is hereditarity of type $A^{\prime}$ apply Theorem 5.1 and Lemma 5.1. If given any three points one cuts between the other two then $M$ is hereditarily unicoherent (Theorem 3.5). Clearly $M$ contains no triods. Thus, by Theorem 5.2, $M$ is hereditarily of type $A^{\prime}$.

\section{REFERENCES}

1. C. E. Capel, Inverse limit spaces, Duke Math. J., 21 (1954), 233-246.

2. H. S. Davis, D. P. Stadtlander and P. M. Swingle, Properties of the set functions $T^{n}$, Portugaliae Mathematica, 21 (1962), 113-133.

3. J. G. Hocking and G. S. Young, Topology, Addison-Wesley, Reading, Mass., 1961.

4. J. K. Kelley, General Topology, Van Nostrand, Princeton, 1955.

5. K. Kuratowski, Topology II, PWN-Academic Press, Warsaw-New York, 1968.

6. S. Mardešić, A locally connected continuum which contains no proper locally connected subcontinuum, Glasnik Matematicki, 2 (22) (1967), 167-178.

7. - Locally connected, ordered and chainable continua, Rad Jugoslav. Akad. Znan. Umjetn., 319 (1960), 147-166.

8. H. C. Miller, On unicoherent continua, Trans. Amer. Math. Soc., 69 (1950), 179194. 
9. R. L. Moore, Foundation of point set theory, Amer. Math. Soc. Colloquium Publications 13, Revised Edition, Providence, 1962.

10. Concerning compact continua which contain no continuum which separates the plane, Proc. Nat. Acad. Sci., U.S.A., 29 (1934), 41-45.

11. E. S. Thomas, Jr., Monotone decompositions of irreducible continua, Rozprawy Matematyczne 50, Warszawa, 1966.

12. L. E. Ward, Jr., Mobs, trees and fixed points, Proc. Amer. Math. Soc., 8 (1957), 798-804.

13. G. T. Whyburn, Analytic Topology, Amer. Math. Soc. Colloquium Publications 28, Providence, 1942.

14. R. L. Wilder, Topology of Manifolds, Amer. Math. Soc. Colloquium Publications 32, Providence, 1949.

Received September 10, 1970. This work was supported by a National Science Foundation Traineeship.

University of CAlifornia, Riverside 


\title{
PACIFIC JOURNAL OF MATHEMATICS
}

\section{EDITORS}

H. SAMELSON

Stanford University

Stanford, California 94305

C. R. HobвY

University of Washington

Seattle, Washington 98105

\section{J. DugunduI}

Department of Mathematics

University of Southern California

Los Angeles, California 90007

RICHARD ARENS

University of California

Los Angeles, California 90024

\section{ASSOCIATE EDITORS}
E. F. BECKENBACH
B. H. Neumann
F. WOLE
K. Yoshida

\section{SUPPORTING INSTITUTIONS}

\author{
UNIVERSITY OF BRITISH COLUMBIA \\ CALIFORNIA INSTITUTE OF TECHNOLOGY \\ UNIVERSITY OF CALIFORNIA \\ MONTANA STATE UNIVERSITY \\ UNIVERSITY OF NEVADA \\ NEW MEXICO STATE UNIVERSITY \\ OREGON STATE UNIVERSITY \\ UNIVERSITY OF OREGON \\ OSAKA UNIVERSITY \\ UNIVERSITY OF SOUTHERN CALIFORNIA
}

\author{
STANFORD UNIVERSITY \\ UNIVERSITY OF TOKYO \\ UNIVERSITY OF UTAH \\ WASHINGTON STATE UNIVERSITY \\ UNIVERSITY OF WASHINGTON

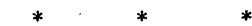 \\ AMERICAN MATHEMATICAL SOCIETY \\ CHEVRON RESEARCH CORPORATION \\ NAVAL WEAPONS CENTER
}

The Supporting Institutions listed above contribute to the cost of publication of this Journal, but they are not owners or publishers and have no responsibility for its content or policies.

Mathematical papers intended for publication in the Pacific Journal of Mathematics should be in typed form or offset-reproduced, (not dittoed), double spaced with large margins. Underline Greek letters in red, German in green, and script in blue. The first paragraph or two must be capable of being used separately as a synopsis of the entire paper. The editorial "we" must not be used in the synopsis, and items of the bibliography should not be cited there unless absolutely necessary, in which case they must be identified by author and Journal, rather than by item number. Manuscripts, in duplicate if possible, may be sent to any one of the four editors. Please classify according to the scheme of Math. Rev. Index to Vol. 39. All other communications to the editors should be addressed to the managing editor, Richard Arens, University of California, Los Angeles, California, 90024.

50 reprints are provided free for each article; additional copies may be obtained at cost in multiples of 50 .

The Pacific Journal of Mathematics is published monthly. Effective with Volume 16 the price per volume (3 numbers) is $\$ 8.00$; single issues, $\$ 3.00$. Special price for current issues to individual faculty members of supporting institutions and to individual members of the American Mathematical Society: $\$ 4.00$ per volume; single issues $\$ 1.50$. Back numbers are available.

Subscriptions, orders for back numbers, and changes of address should be sent to Pacific Journal of Mathematics, 103 Highland Boulevard, Berkeley, California, 94708.

PUBLISHED BY PACIFIC JOURNAL OF MATHEMATICS, A NON-PROFIT CORPORATION

Printed at Kokusai Bunken Insatsusha (International Academic Printing Co., Ltd.), 7-17, Fujimi 2-chome, Chiyoda-ku, Tokyo, Japan. 


\section{Pacific Journal of Mathematics}

\section{Vol. 36, No. $3 \quad$ BadMonth, 1971}

E. M. Alfsen and B. Hirsberg, On dominated extensions in linear subspaces of

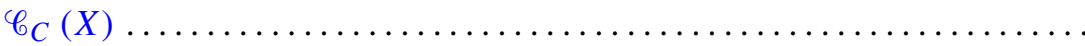

Joby Milo Anthony, Topologies for quotient fields of commutative integral

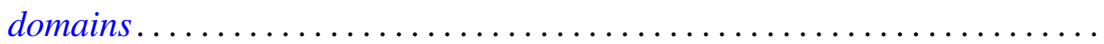

V. Balakrishnan, G. Sankaranarayanan and C. Suyambulingom, Ordered cycle lengths in a random permutation .......................... 603

Victor Allen Belfi, Nontangential homotopy equivalences............... 615

Jane Maxwell Day, Compact semigroups with square roots .............. 623

Norman Henry Eggert, Jr., Quasi regular groups of finite commutative nilpotent algebras .......................................... 631

Paul Erdős and Ernst Gabor Straus, Some number theoretic results ......... 635

George Rudolph Gordh, Jr., Monotone decompositions of irreducible Hausdorff continua .............................................

Darald Joe Hartfiel, The matrix equation $A X B=X \ldots \ldots \ldots \ldots \ldots \ldots . \ldots 69$

James Howard Hedlund, Expansive automorphisms of Banach spaces. II . . . . 671

I. Martin (Irving) Isaacs, The p-parts of character degrees in p-solvable

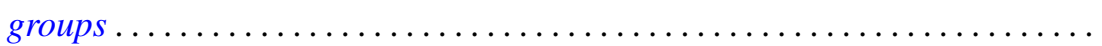

Donald Glen Johnson, Rings of quotients of $\Phi$-algebras ............... 693

Norman Lloyd Johnson, Transition planes constructed from semifield planes....

Anne Bramble Searle Koehler, Quasi-projective and quasi-injective modules.

James J. Kuzmanovich, Completions of Dedekind prime rings as second endomorphism rings...

B. T. Y. Kwee, On generalized translated quasi-Cesàro summability ...

Yves A. Lequain, Differential simplicity and complete integral closure

741

Mordechai Lewin, On nonnegative matrices.

753

Kevin Mor McCrimmon, Speciality of quadratic Jordan algebras ...

Hussain Sayid Nur, Singular perturbations of differential equations in abstract spaces .....................................

D. K. Oates, A non-compact Krein-Milman theorem .

Lavon Barry Page, Operators that commute with a unilateral shift on an invariant subspace...

Helga Schirmer, Properties of fixed point sets on dendrites.

Saharon Shelah, On the number of non-almost isomorphic models of $T$ in a

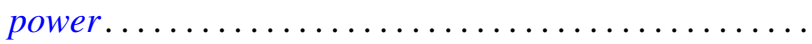

Robert Moffatt Stephenson Jr., Minimal first countable Hausdorff spaces....

Masamichi Takesaki, The quotient algebra of a finite von Neumann algebra 